\title{
Retrospective evaluation of surgical outcomes after closure of durotomy in eight dogs affected by spinal subarachnoid diverticulum
}

\author{
Simone Spinillo ${ }^{1 *}$, Lorenzo Golini ${ }^{1,2}$, Massimo Mariscoli ${ }^{1,3}$ and Luca Motta \\ ${ }^{1}$ Northwest Veterinary Specialists, Runcorn, UK \\ ${ }^{2}$ Vetsuisse Faculty, University of Zürich, Zürich, Switzerland \\ ${ }^{3}$ Paragon Veterinary Referrals, Wakefield, UK
}

\begin{abstract}
Background: Canine spinal subarachnoid diverticulum has been studied since 1968 and a few advancements have been made with regard to the treatment of this disease. Several surgical techniques have been proposed which include durectomy, fenestration, and/or marsupilization with the latter two regarded as the more favorable surgical treatments. Aim: In this retrospective study, we evaluated the closure of the durotomy incision in the treatment of canine spinal subarachnoid diverticulum.

Methods: We retrospectively evaluated eight dogs with clinical signs and magnetic resonance imaging findings consistent with spinal subarachnoid diverticulum located in the cervical and thoracolumbar area. This was to determine whether the fenestration with closure of durotomy was superior to the marsupialization technique. In all dogs, fenestration and removal of subarachnoid adherences alongside closure of the durotomy were carried out.

Results: Mild post-operative deterioration was observed in all dogs. Follow-up from 7 to 36 months indicated a satisfactory outcome.

Conclusion: We conclude that the closure of durotomy has similar short-term and long-term outcomes compared to the previous reported studies. These results suggest that the closure of durotomy is a viable technique for spinal subarachnoid diverticulum.
\end{abstract}

Keywords: Arachnoid cyst, Durotomy, Spinal cord, Subarachnoid cyst, Subarachnoid diverticulum.

\section{Introduction}

Spinal arachnoid diverticulum (SAD) is a dilatation of the subarachnoid space filled by cerebrospinal fluid (CSF) that can occur at any level of the spinal cord (Skeen et al., 2003). Over the past years, the novelty of this disease has aroused interest leading to the publication of many articles. However, this has proven the need for clarification as this condition has been referred to by different names, including subarachnoid cyst, meningeal cyst, leptomeningeal cyst/pseudocyst, arachnoid cyst, intra-arachnoid cyst. The term "cyst" has been inappropriately used to describe the "leptomeningeal cavitation" or "dilatation" of the subarachnoid space (Gnirs et al., 2003). The reason being that histologically it is lacking a true epithelial lining (Lowrie et al., 2014). The term diverticulum or dilatation is therefore preferred as it denotes a more accurate description. The term SAD will be used in this article. The pathophysiology of the development of canine SAD remains unknown. Congenital and acquired theories have been proposed including the abnormal development of the arachnoid architecture, alteration of the one-way valve CSF flow, inflammation of the spinal cord, spinal cord trauma, caudal articular process dysplasia, and intervertebral disk disease. A causal relationship between these theories and the development of the SAD has not yet been confirmed. (Dyce et al., 1991; McKee and Renwick, 1994; Frykman, 1999; Webb, 1999; Skeen et al., 2003; Lowrie et al., 2014; Driver et al., 2019; Rohdin et al., 2020). SAD is often localized at the cervical region in large dog breeds and in the thoracolumbar spinal cord in small breeds (Jurina and Grevel, 2004; Mauler et al., 2014, 2017). Pugs, Rottweilers, and French bulldogs are the overrepresented breeds (Jurina and Grevel, 2004; Mauler et al., 2014). The reported clinical presentation is characterized by slow progressive general proprioceptive ataxia and paraparesis with or without fecal and urinary incontinence and absence of hyperesthesia (Mauler et al., 2014). Medical and surgical treatments have been compared and supporting surgical management leads to a better outcome (McKee and Renwick, 1994; Frykman, 1999; Skeen et al., 2003; Mauler et al., 2017). The surgical approaches previously described are either by dorsal laminectomy or hemilaminectomy according to the SAD location. Surgical techniques already described vary from durotomy, durotomy with marsupialization, and partial durectomy, all with removal of the adherences to reduce spinal cord compression and avoiding the recurrence of the SAD (Ness, 1998; Frykman, 1999; Gnirs et al., 2003; Skeen et al., 2003; Jurina and Grevel, 2004). 
Relapse of the clinical signs after surgery is common (Alisauskaite et al., 2019), but it is not always related to the regrowth of the SAD (Alcoverro et al., 2018). In dogs, relapse can occur between 7 months and 4 years postoperatively (Oxley and Pink, 2012; Bismuth et al., 2014; Alcoverro et al., 2018) and the long-term prognosis in pugs is poor (Alisauskaite et al., 2019). The purpose of this retrospective study was to describe the surgical treatment consisting of fenestration and removal of subarachnoid adherences, alongside closure of the durotomy and to report the short-term and long-term outcomes.

\section{Materials and Methods}

The medical and surgical records of eight dogs affected by SAD and treated with the same surgical technique at Northwest Veterinary Specialists (Sutton Weaver, Asheville point, Runcorn, UK) and Neurovet (Legnano, Italy) between April 2017 and April 2020 were identified and retrospectively reviewed.

The following data were recorded for each dog: signalment, clinical history, clinical and neurological examinations, magnetic resonance imaging (MRI) findings, confirmed diagnosis after surgical exploration, standardized surgical procedure, and outcome at least 6 months after surgery. Dogs were anaesthetized according to the standard ethics procedure using anesthetic medications. MRI of the spinal cord was acquired under general anesthesia in dorsal recumbency using low-field MRI scanners (Hitachi aperto grande 0.4T; Esaote Vet Grande, Genova, Italy). Interpretation of the images was obtained in seven dogs by a board-certified radiologist and in one dog by a neurology clinician. All spinal surgeries were performed using a surgical microscope (Zeiss NC-2). A dorsal laminectomy approach was taken in all cases by removing the dorsal lamina over the SAD and preserving the articular facets joints (Funkquest, type B). This approach permitted a good visualization of the dorsal spinal cord, allowing the performance of a longitudinal durotomy with a no. 11 blade and microsurgical Castroviejo scissors (Fig. 1). The durotomy was extended cranially and caudally to the SAD until normal spinal cord was exposed. Stay sutures were then applied at the dural edges and maintained in place with small hemostats using a polydioxanone 6-0 (Ethicon LLC, San Lorenzo, PR) (Fig. 2). This technique exposed the SAD allowing the visualization of the subarachnoid diverticulum, subarachnoid CSF accumulation, and surrounding adhesions, most of which were microsurgically removed (Figs. 3-5). The stay sutures were not used for dural closure, and the holes of the stay sutures were incorporated into the durotomy repair. In all cases, the dural closure was completed using a simple continuous pattern with polydioxanone 6-0 (Ethicon LLC, San Lorenzo, PR) in seven dogs and with polyamide 5-0 (Ethicon LLC, San Lorenzo, PR) in one dog; waterproof suture was achieved by noting the absence of CSF leakage (Fig. 6). No dural patch or autologous

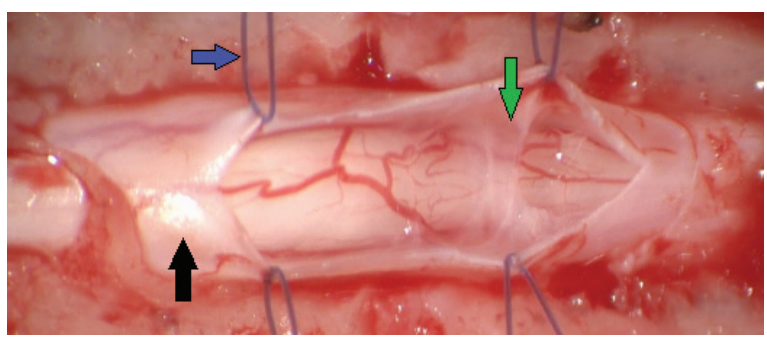

Fig. 1. Spinal cord (black arrow) after longitudinal durotomy and adherences exposure (green arrow) via stay suture (blue arrow).

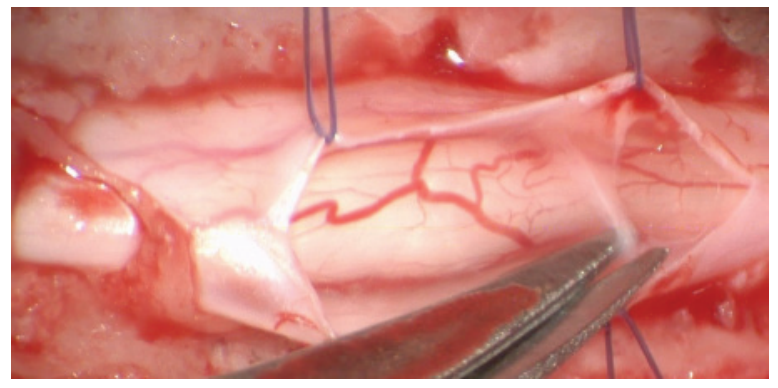

Fig. 2. Stay suture in place and dorsal removal of the spinal subarachnoid adherences with Castroviejo scissors.

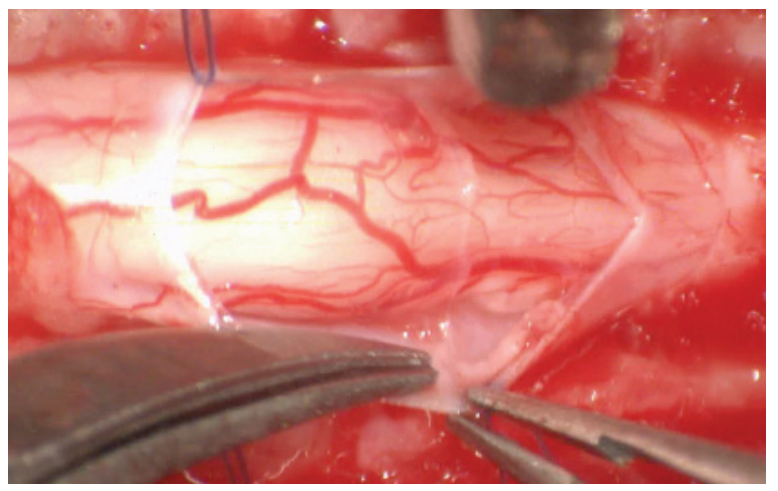

Fig. 3. Stay suture in place and lateral removal of the spinal subarachnoid adherences with Castroviejo scissors.

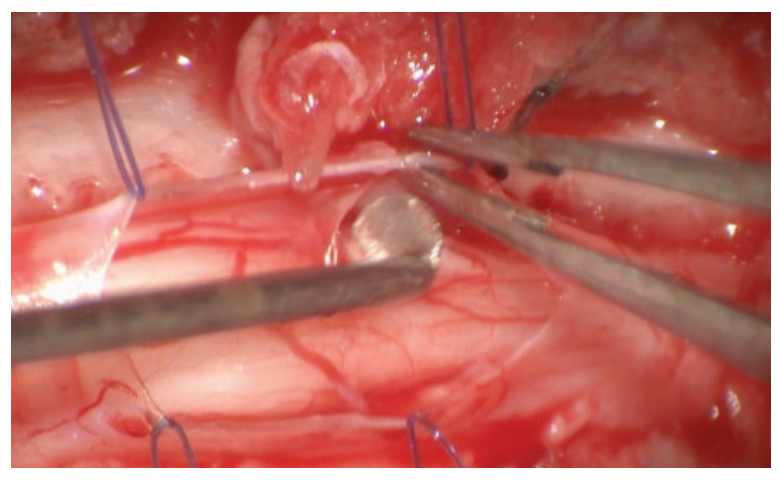

Fig. 4. Removal of the surrounding adhesion. 


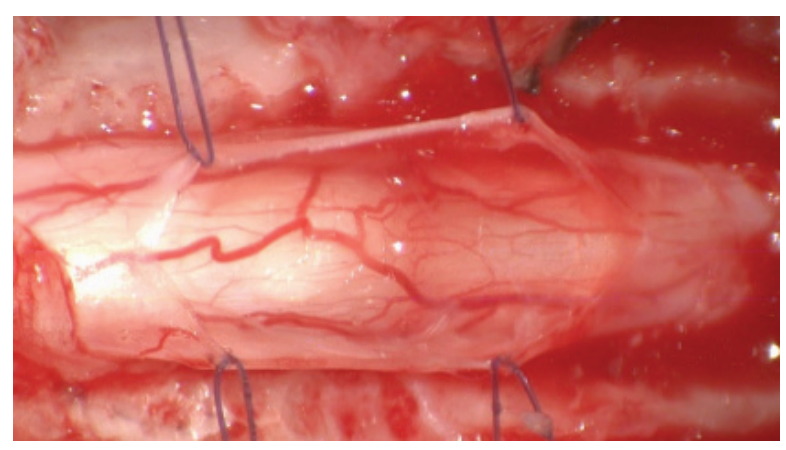

Fig. 5. Spinal cord free form adherences and CSF flow restored.

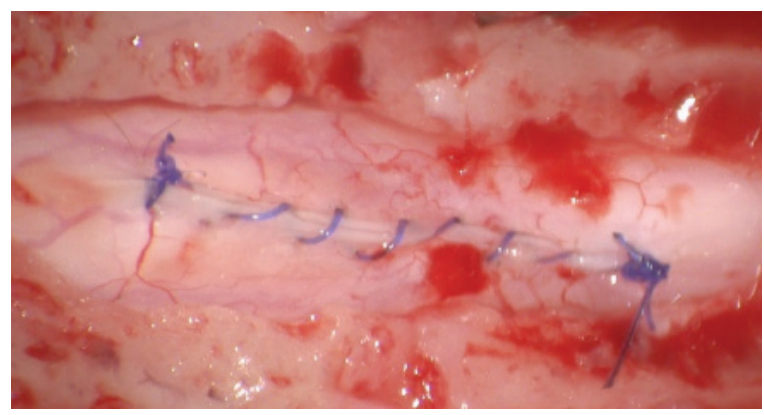

Fig. 6. Closure of the durotomy.

material was placed over the laminectomy defect. The muscular, subcutaneous, and skin layers were routinely closed. The Glasgow modified pain score system was utilized for the postoperative provision of analgesia. Dogs were hospitalized until good pain management was achieved with oral medication and satisfactory movements of pelvic limbs were observed. Outcome was deemed unsuccessful if there was progressive neurological deterioration and successful if the neurological signs were similar or improved compared to the neurological signs at presentation. Short-term and long-term outcomes were defined as less than 7 months and more than 7 months. Followups were evaluated by neurological examination by the authors in the same institution where the spinal surgery was performed or by custom-designed telephone questionnaire used by Alisauskaite et al. (2019), along with a video evaluation of the dog sent via email from the owner. In addition, owner's level of satisfaction regarding the neurological improvement was recorded.

\section{Ethical approval}

Ethics committee approval for this study was not sought because this retrospective study was based on the analysis of the already acquired data. Informed written consent to use and share data was obtained from each owner.

\section{Results}

Eight dogs met the inclusion criteria: four Pugs, two Rottweilers, and two French Bulldogs. All eight dogs were male, of which two were neutered and six were entire. Median age at onset of clinical signs was 36 months (ranging from 7 to 108 months). Neurological signs were chronic progressive ambulatory paraparesis and hind limbs ataxia (dogs 1-4 and 7) and ambulatory tetraparesis with general proprioceptive ataxia and fore limbs hypermetria (dogs 5, 6, and 8). Dog 4 presented with subacute onset of ambulatory paraparesis and general proprioceptive ataxia. Mean duration of the neurological signs before the presentation was 13 weeks (ranging from 1 week to 12 months). All dogs were urinary and fecal continent with no signs of paraspinal discomfort (Table 1). MRI was carried out according to the neuroanatomical localization in all dogs and SAD was diagnosed in the cervical spinal cord in three dogs (dogs 5, 6, and 8) and in the thoracolumbar region in five dogs (dogs 1-4 and 7). In six out of eight dogs, the MRI showed concurrent imaging findings in proximity of the SAD including vertebral malformations, spondylosis deformans, minimal to marked intervertebral disk protrusion, and intramedullary T2-W hyperintensity extending cranially or caudally to the cervical SAD (Table 2). SAD localization was dorsal or dorsolateral with various degree of spinal cord compression detected in cross-sectional images. After the completion of the dorsal laminectomy, enlargement of the spinal cord at the level of the SAD was visualized in all dogs. Durotomy resulted in a clear CSF leakage. Intraoperative microscopic findings after the placement of stay sutures included thickening of the dura mater in the location of the SAD and subarachnoid "band-like" adhesions causing an "hourglass" shape of the spinal cord and adhesions to arterial blood vessels. Most of the subarachnoid adhesions were microsurgically removed until CSF flow was reestablished, but some adhesions were not removed to avoid spinal cord damage and hemorrhage. Intraoperative bleeding of the dural and pial vessels was controlled with collagen hemostat (Lyostypt, BBraun, Sheffield, UK). Edema of the spinal cord was observed before the closure of the dura; however, this did not impede the latter procedure and did not result in iatrogenic constriction of the spinal cord. The dura was then closed with polydioxanone 6.0 or polyamide $5-0$ in a continued pattern. No CSF leakage through the dural closure was visible at the end of the procedure. Histopathological analysis of the adhesions was carried out in one $\operatorname{dog}(\operatorname{dog} 7)$ and it revealed a spindle cell proliferation with collagen deposit consistent with fibrosis. There was no evidence of inflammation or malignant cells. Postoperatively, all dogs were hospitalized for a few days for cage rest and medical treatment. Median postoperative hospitalization time was 5 days (3-8 days). In all dogs, 


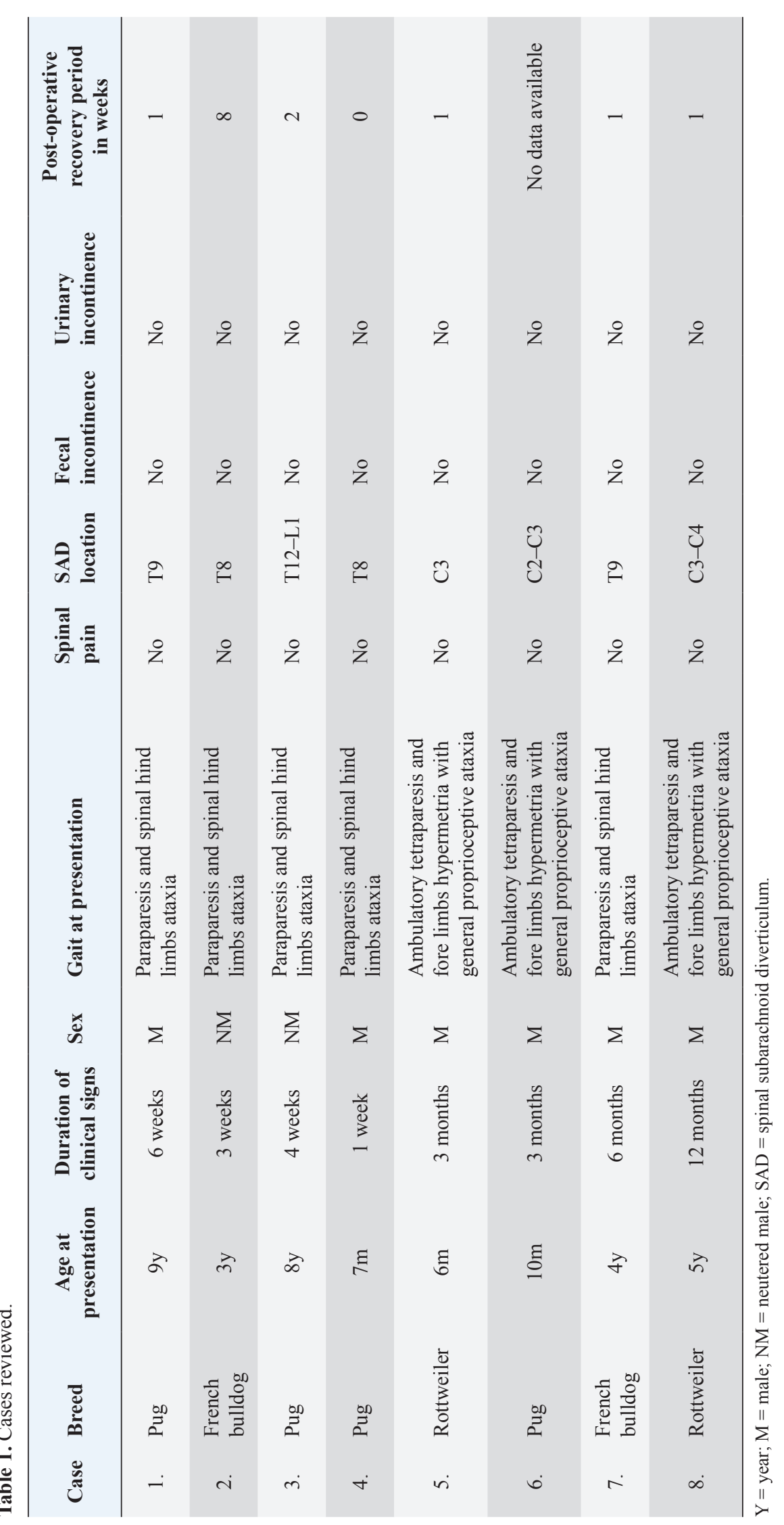


Table 2. MRI findings.

\begin{tabular}{|c|c|c|c|}
\hline Case & Breed & SAD location & Concurrent diagnostic imaging findings \\
\hline 1. & Pug & $\begin{array}{l}\text { Dorsal SAD at T9 tapering caudally } \\
\text { at cranial } \mathrm{T} 10.25 \% \text { dorsal compression } \\
\text { at mid } \mathrm{T} 9\end{array}$ & Mild kyphosis T8/T9 and mild spondylosis deformans. \\
\hline 2. & $\begin{array}{l}\text { French } \\
\text { bulldog }\end{array}$ & $\begin{array}{l}\text { Dorsolateral (right) SAD caudally to } \\
\text { T8 with } 50 \% \text { spinal cord compression. }\end{array}$ & $\begin{array}{l}\text { Congenital malformation of T8 (shorter than normal) and } \\
\text { T9 (dorsal hemivertebrae leading to scoliosis and marked } \\
\text { kyphosis. Chronic intervertebral disc protrusions and variable } \\
\text { degrees of spinal cord compression at T8-T9 and T9-T10. } \\
\text { SAD at T8. Syringohydromyelia extending cranially from T8. }\end{array}$ \\
\hline 3. & Pug & $\begin{array}{l}\text { Dorsolateral (left) SAD extending } \\
\text { from T12 up to cranially to L1. }\end{array}$ & $\begin{array}{l}\mathrm{T} 11-\mathrm{T} 12 \text { articular facet hypoplasia, } \mathrm{T} 13-\mathrm{L} 1 \text { mild disc } \\
\text { protrusion. Increased } \mathrm{T} 2-\mathrm{W} \text { hyperintensity intramedullary } \\
\text { along } \mathrm{T} 12-\mathrm{L} 1 .\end{array}$ \\
\hline 4. & Pug & $\begin{array}{l}\text { Dorsal bilobed } \mathrm{SAD} \text { at } \mathrm{T} 8 \text { tapering } \\
\text { cranially with } 25 \% \text { reduction of } \\
\text { spinal cord. }\end{array}$ & $\begin{array}{l}\text { Mild disc protrusion } \mathrm{T} 8 / \mathrm{T} 9 \text { and } \mathrm{T} 9 / \mathrm{T} 10 \text { circa } 10 \% \text { minimal } \\
\text { reduction of the spinal cord and no compression. }\end{array}$ \\
\hline 5. & Rottweiler & $\begin{array}{l}\text { Dorsal symmetrical SAD caudally to } \\
\text { C2 up to cranial C } 3 \text { tapering cranially } \\
\text { with marked SC compression and SC } \\
\text { diameter reduction by } 50 \% \text {. Marked SC } \\
\text { compression. }\end{array}$ & Syringohydromyelia extending caudally up to $\mathrm{C} 4$ \\
\hline 6. & Pug & $\begin{array}{l}\text { Focal dorsal symmetrical SAD mid } \\
\mathrm{C} 2 \text { to mid } \mathrm{C} 3 \text { moderate spinal cord } \\
\text { compression. }\end{array}$ & Syringohydromyelia extending caudally up to C6 \\
\hline 7. & $\begin{array}{l}\text { French } \\
\text { bulldog }\end{array}$ & $\begin{array}{l}\text { Left dorsal aspect of the SC at mid T9 } \\
\text { tapering cranially with marked spinal cord } \\
\text { compression. }\end{array}$ & None. \\
\hline 8. & Rottweiler & $\begin{array}{l}\text { Dorsolateral (left) SAD extending } \\
\text { caudally to mid C2 up to cranial C3 } \\
\text { tapering cranially with marked SC } \\
\text { compression and SC diameter reduction } \\
\text { by } 50 \% \text {. Marked spinal cord compression. }\end{array}$ & None. \\
\hline
\end{tabular}

there was an initial postoperative deterioration ranging from a worsening of weakness to non-ambulatory paraparesis, followed by a gradual improvement which occurred between 1 and 8 weeks postoperatively. Short-term outcome at 7 months was available for all dogs. Six out of eight dogs had a successful short-term outcome. Two dogs (dogs 2 and 3 ) had unsuccessful outcomes with progressive deterioration starting at 3 and 7 months post-surgery characterized by fecal and urinary incontinence and non-ambulatory paraparesis. Dog 2 had a follow-up MRI that detected recurrence of SAD and cranial extension of the intramedullary T2W hyperintensity. In this case, medical treatment was attempted with corticosteroid at $0.5 \mathrm{mg} / \mathrm{kg}$ once daily for 30 days without improvement of the neurological status over 7 months after repeat MRI. Dog 2 deceased 12 months after surgery and $\operatorname{dog} 3$ deceased 6 months after surgery, both being from causes unrelated to the neurological condition. Long-term outcome longer than 7 months (from 7 to 36 months; median 24 months) was available for six dogs. All dogs after the initial post-surgical improvement stayed in a static neurological condition characterized by general proprioceptive ataxia, ambulatory tetra/paraparesis, and urinary/fecal continence. All the owners were satisfied with both the surgical outcomes and the dogs' quality of life. Telephone questionnaires were obtained in cases $1,6,7$, and 8. The owners of cases 6 and 7 provided video footage of the dogs' gait. Cases 4 and 5 were examined by the primary clinician. Case 4 was spastic ambulatory paraparetic with moderate general proprioceptive ataxia and delayed proprioception in the hind limbs. Case 5 was ambulatory tetraparetic with general proprioceptive ataxia and postural reactions delayed in all four limbs. Four months postsurgery, case 7 experienced an episode of acute hind limbs weakness and the referring veterinary surgeon prescribed corticosteroid at $0.25 \mathrm{mg} / \mathrm{kg}$ once daily. At the time of writing this article, the dog had experienced no worsening of the gait. In all the patients, there was minimal scuffing of the paws; however, this did worsen with increased exercise time. 


\section{Discussion}

We have retrospectively described a surgical technique and outcome for the treatment of SAD consisting of durotomy closure after removal of the leptomeningeal adhesion. Our results support a satisfactory long-term outcome. All the dogs in this study were male and this is in agreement with previous studies which suggested a male predisposition for the SAD (Jurina and Grevel, 2004; Flegel et al., 2013; Mauler et al., 2014; Rohdin et al., 2014; Alcoverro et al., 2018). The dog's age range from 6 months to 9 years with the majority of the dogs presented with progressive gait alteration and only one dog presented with a subacute onset. There was no correlation between age and duration of clinical signs, and the latter did not affect the postoperative recovery period or the surgical outcome. There is a disagreement in the literature in relation to the long-term outcome in dogs affected by SAD. Some authors describe that $86 \%$ of the pugs have poor long-term post-surgical outcome (12 months) (Alisauskaite et al., 2019), whereas other researchers describe fair long-term outcome (23 months) in $82 \%$ of dogs (Mauler et al., 2017). In our study, the majority of the dogs were pugs (4 out of 8) and $75 \%$ of them had a successful long-term outcome, with a median follow-up of 21 months. Three dogs (one French bulldog and two Rottweilers) had successful outcomes, with a median follow-up of 24 months. Two dogs (one Pug and one French bulldog) deteriorated in third and seventh month postoperatively.

All dogs in the study experienced a post-surgical deterioration varying from mild deterioration of the gait to non-ambulatory paraparesis. The post-operative recovery period ranged from 1 to 8 weeks with four out of seven dogs recovering within 1 week. Interestingly, the only two dogs with longer post-operative recovery period eventually deteriorated in the short-term followup (mean: 5 months). In large breed dogs, the SAD is commonly localized at the cervical region and this is in agreement with our findings. In addition in our study, the long-term outcome was successful as previously described in other studies (Gnirs et al., 2003; Jurina and Grevel, 2004; Mauler et al., 2014).

This is the first retrospective study in which the overall short-term and long-term outcomes are recorded in different breed of dogs using the same surgical technique. Previous studies compared the short-term and long-term outcomes based on different surgical techniques (Skeen et al., 2003; Jurina and Grevel, 2004; Bismuth et al., 2014; Mauler et al., 2017; Alisauskaite et al., 2019). The innovation of this original surgical technique is based on the fact that the closure of the durotomy defect was carried out in all cases.

Achieving watertight dural closure can be difficult in dogs due to the small laminectomy approach, the narrow space, and the depth of the surgical field, which make the manipulation of microsurgical instruments challenging. However, even in view of these challenges the closure of the dura mater can be carried out. The rationale behind the decision of closing the dura was the belief of the authors that the SAD formation is due to the subarachnoid adherences that in turn allows the dilatation of the subarachnoid space. Based on this hypothesis, removal of the adhesions would allow normalization of the CSF flow which without closure of the durotomy, would freely bath the surrounding soft tissues potentially predisposing to pseudomeningocele. We also believe that the closure of the durotomy does not avoid the formation of the dorsal laminectomy membrane, but it avoids the direct contact of the surrounding soft tissues to the exposed spinal cord and a potential spinal cord herniation.

A recent study suggested that the deterioration of clinical signs after SAD surgery could occur in absence of SAD reformation (Alcoverro et al., 2018). Only one of the two dogs that deteriorated in the short-term period had a repeat MRI scan which detected the reformation of the SAD at the same level of the previous SAD. It is unknown whether the neurological deterioration of the other dog was caused by a recurrence of the SAD or by other spinal pathologies. In the successful surgical cases, the MRI scan was not repeated as the dogs, after initial improvement, either did not deteriorate or progressively improved.

In humans, intradural arachnoid cysts are a similar condition to the canine SAD and it can be congenital or acquired (Osenbach et al., 1992; Silbergleit et al., 1998). Humans may experience discomfort and various degrees of neurological dysfunction. Surgical fenestration for removal of the adhesions or cysts and closure of durotomy can lead to full recovery (French et al., 2017). However, there are a significant number of patients who can experience no changes in clinical signs even in absence of cyst recurrence (Osenbach et al., 1992). In addition, 30\% of the patients who present with ataxia may fail to recover a normal gait after surgery (French et al., 2017). Another disease in humans that seems similar to canine SAD is the spinal adhesive arachnoiditis, a rare spinal disease that is commonly caused by tuberculosis, syphilis, meningitis, spinal cord neoplasm, and injection of oil-based contrast material during myelographic studies (Dolan, 1993). Surgically, the durotomy defect is either closed routinely or a dural substitute is sewed to the dura. The prognosis is poor and surgical intervention may provide only temporary relief (Wright and Denney, 2003).

Little is known about the most appropriate medical therapy for canine SAD; the role of anti-inflammatory medications is largely unknown. Prednisolone was administered to $\operatorname{dog} 7$ that presented to the referring veterinary surgeons with acute hind limbs weakness, which largely resolved. The owner was unwilling to discontinue the corticosteroid therapy and, therefore, it remains unknown whether the dog would have experienced a sustained improvement even without the use of anti-inflammatory therapy. All dogs did not become neurologically normal and a various degree 
of ataxia and hind limbs weakness were present in the long-term follow-up. A common report from owners was toe nail scuffing that worsened after long exercise activity. Despite this, all the owners were satisfied with the outcome and with the dogs' quality of life.

The first limitation of this study is the retrospective nature of it and the small number of cases. Another limitation is that in some cases, the assessment of the dogs' neurological status was carried out by a telephone-based questionnaire and not by a completed neurological examination carried out by the clinician. However, this assessment method has largely been used (Gnirs et al., 2003; Mauler et al., 2017; Alisauskaite et al., 2019) and it was deemed exhaustive for the purpose of this study. To the authors' knowledge, this is the first retrospective study that describes an original surgical technique for the treatment of SAD consisting of closure of the durotomy after removal of the leptomeningeal adhesion with favorable short-term and long-term outcomes.

\section{Acknowledgments}

We thank Dr. Daniele Corlazzoli (CVRS, Policlinico Veterinario Roma Sud, Rome, Italy) for providing the original idea for the surgical technique described in this article. We also thank Dr. Alberto Cauduro (Neurovet, via Maestri del Lavoro 29 Legnano, Milano, Italy) for providing a case (case n. 8) for this study.

\section{Conflict of interest}

The authors declare that there is no conflict of interest. Authors'contribution

All authors conceived the study, wrote the paper, and were involved in the clinical outcome follow-up. All authors read and approved the final manuscript.

\section{References}

Alcoverro, E., McConnell, J.F., Sanchez-Masian, D., De Risio, L., De Decker, S. and Gonçalves, R. 2018. Late-onset recurrence of neurological deficits after surgery for spinal arachnoid diverticula. Vet. Rec. $182,380$.

Alisauskaite, N., Cizinauskas, S., Jeserevics, J., Rakauskas, M., Cherubini, G.B., Anttila, M. and Steffen, F. 2019. Short- and long-term outcome and magnetic resonance imaging findings after surgical treatment of thoracolumbar spinal arachnoid diverticula in 25 Pugs. J. Vet. Intern. Med. 33, 1376-1383.

Bismuth, C., Ferrand, F.X., Millet, M., Buttin, P., Fau, D., Cachon, T., Viguier E., Escriou C. and Carozzo, C. 2014. Original surgical treatment of thoracolumbar subarachnoid cysts in six chondrodystrophic dogs. Acta Vet. Scand. 56, 32.

Dolan, R.A. 1993. Spinal adhesive arachnoiditis. Surg. Neurol. 39, 479-484.

Driver, C.J., Rose, J., Tauro, A., Fernandes, R. and Rusbridge, C. 2019. Magnetic resonance image findings in pug dogs with thoracolumbar myelopathy and concurrent caudal articular process dysplasia. BMC Vet. Res. 15, 182.

Dyce, J., Heritage, M.E., Houlton, J.E.F. and Palmer, A.C. 1991. Canine spinal 'arachnoid cysts'. J. Small Anim. Pract. 32, 433-437.

Flegel, T., Müller, M.K., Truar, K., Löffler, C. and Oechtering, G. 2013. Thoracolumbar spinal arachnoid diverticula in 5 pug dogs. Can. Vet. J. 54, 969-973.

French, H., Somasundaram, A., Biggs, M., Parkinson, J., Allan, R., Ball, J., and Little, N. 2017. Idiopathic intradural dorsal thoracic arachnoid cysts: A case series and review of the literature. J. Clin. Neurosci. 40, 147-152.

Frykman, F. 1999. Spinal arachnoid cyst in four dogs: diagnosis, surgical treatment and follow-up results. J. Small Anim. Pract. 40, 544-549.

Gnirs, K., Ruel, Y., Blot, S., Begon, D., Rault, D., Delisle, F., Boulouha, L., Colle, M., Carozzo, C. and Moissonnier, P. 2003. Spinal subarachnoid cysts in 13 dogs. Vet. Radiol. Ultrasound. 44, 402-408.

Jurina, K. and Grevel, V. 2004. Spinal arachnoid pseudocysts in 10 rottweilers. J. Small Anim. Pract. $45,9-15$.

Lowrie, M.L., Platt, S.R. and Garosi, L.S. 2014. Extramedullary spinal cysts in dogs. Vet. Surg. 43, 650-662.

Mauler, D.A., De Decker, S., De Risio, L., Volk, H.A., Dennis, R., Gielen, I., Van der Vekens, E., Goethals, K. and Van Ham, L. 2014. Signalment, clinical presentation, and diagnostic findings in 122 dogs with spinal arachnoid diverticula. J. Vet. Intern. Med. 28, 175-181.

Mauler, D.A., De Decker, S., De Risio, L., Volk, H.A., Dennis, R., Gielen, I., Van der Vekens, E., Goethals, K. and Van Ham, L. 2017. Spinal arachnoid diverticula: outcome in 96 medically or surgically treated dogs. J. Vet. Intern. Med. 31, 849-853.

McKee, W.M. and Renwick, P.W. 1994. Marsupialisation of an arachnoid cyst in a dog. J. Small Anim. Pract. 35, 108-111.

Ness, M.G. 1998. Spinal arachnoid cysts in two shih tzu littermates. Vet. Rec. 142, 515-516.

Osenbach, R.K., Godersky, J.C., Traynelis, V.C. and Schelper, R.D. 1992. Intradural extramedullary cysts of the spinal canal: clinical presentation, radiographic diagnosis, and surgical management. Neurosurgery. 30, 35.

Oxley, W. and Pink, J. 2012. Amelioration of caudal thoracic syringohydromyelia following surgical management of an adjacent arachnoid cyst. J. Small Anim. Pract. 53, 67-72.

Rohdin, C., Ljungvall, I., Häggström, J., Leijon, A., Lindblad-Toh, K., Matiasek, K., Rosati, M., Wohlsein, P. and Jaderlund, K.H. 2020. Thoracolumbar meningeal fibrosis in pugs. J. Vet. Intern. Med. 34, 797-807. 
Rohdin, C., Nyman, H.T., Wohlsein, P. and Hultin Jäderlund, K. 2014. Cervical spinal intradural arachnoid cysts in related, young pugs. J. Small Anim. Pract. 55, 229-234.

Silbergleit, R., Brunberg, J.A., Patel, S.C., Mehta, B.A. and Aravapalli, S.R. 1998. Imaging of spinal intradural arachnoid cysts: MRI, myelography and CT. Neuroradiol. 40, 664-668.
Skeen, T.M., Olby, N.J., Muñana, K.R. and Sharp, N.J. 2003. Spinal arachnoid cysts in 17 dogs. J. Am. Anim. Hosp. Assoc. 39, 271-282.

Webb, A.A. 1999. Intradural spinal arachnoid cyst in a dog. Can. Vet. J. 40, 588-589.

Wright, M.H. and Denney, L.C. 2003. A comprehensive review of spinal arachnoiditis. Orthop. Nurs. 22, 262. 